\title{
Des similitudes intéressantes
}

\section{Jacques Cornuz}

Professeur, Directeur, médecin chef, Policlinique médicale universitaire (PMU) et Chef du département universitaire de médecine et santé communautaire CHUV, Lausanne

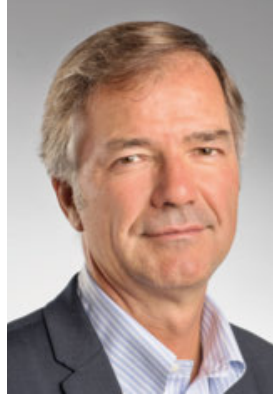

L'agenda du dernier congrès annuel des généralistes français (Paris, Palais de Congrès, 5-7 avril 2018) mérite que l'on s'y arrête quelques minutes (www.congresmg. $\mathrm{fr} / \mathrm{fr} /$ ). Voyez plutôt les thèmes mis en exergue par le comité scientifique: 1) la médecine dans le débat public; 2) la pluriprofessionnalité; 3) les autres disciplines et les institutions de santé; 4) l'implication des patients et la décision partagée.

Les similitudes avec les enjeux de la médecine en Suisse sont frappantes.

En premier lieu, le débat public. Les généralistes savent que la profession médicale est l'une des professions les plus respectées dans nos sociétés occidentales: notre avis est apprécié, nos prises de position comptent, notre poids dans le débat public est important. Pour la santé de la population, nous devons nous engager, faire acte de plaidoyer, d'«advocacy». A ce sujet, le soutien clair et sans ambiguïté de la FMH à l'initiative populaire fédérale «Oui à la protection des enfants et des jeunes contre la publicité pour le tabac» est un bel exemple d'engagement professionnel au service de la santé des citoyens et citoyennes suisses.

Deuxièmement, l'interprofessionnalité. Dans ce domaine, la Suisse est regardée avec beaucoup d'intérêt. Il est en effet fort intéressant de constater à quel point nos collègues français sont interpellés par le rôle des assistantes médicales des cabinets médicaux en Suisse. $\mathrm{Au}$ point que leur président va prochainement étudier cette particularité suisse afin d'évaluer dans quelle mesure ce modèle peut être "transféré» en France! Ils perçoivent la plus-value de cette complémentarité professionnelle au bénéfice de la santé de notre population et d'une prise en charge optimale des patients avec maladie chronique.

Troisièmement, les liens avec les autres disciplines médicales et institutions de santé. Eux aussi ont perçu à quel point la médecine générale doit faire des ponts avec les autres disciplines. Se faire reconnaître comme une spécialité ne doit pas empêcher les généralistes de favoriser une approche transversale de la médecine et des soins. La jeune génération des généralistes français, très présente au Congrès, semble particulièrement sensible à cette approche.

Quatrièmement, la participation des patients en vue d'un processus de décision partagée. Nos collègues français ont décidé de s'interroger sur le rôle des patients dans le processus décisionnel. Emblématiquement, et judicieusement à mes yeux, les enjeux du dépistage ont permis d'aborder cette thématique. Pour rappel, un dépistage apparaît toujours plus attractif qu'il ne l'est en vérité, ceci autant aux médecins qu'aux patients: avancement du diagnostic sans modification du pronostic (lead time); identification de tumeurs indolentes, qui n'auraient jamais été symptomatiques (overdiagnosis); bénéfice souvent exprimé en réduction du risque relatif, donc de manière plus avantageuse qu'en réduction du risque absolu (number needed to be screened); image du médecin qui «s'occupe bien de ses patients", non seulement quand ils sont malades, mais également de manière proactive. Nos collègues français mettent également en avant le biais du regret anticipé, cette crainte de regretter, a posteriori, une fois le cancer déclaré, de ne pas avoir anticipé la maladie à un stade plus précoce. Il a alors été rappelé l'importance de demander au patient le rôle qu'il souhaite prendre dans le processus décisionnel. A celles et à ceux qui nous disent «Docteur, c'est vous qui savez, vous êtes docteur!», l'attitude sera peut-être différente de celle que nous adoptons avec les patients qui précisent: «Et bien, puisque vous m'en offrez la possibilité, je prends volontiers le temps de peser avec vous les 〈pour» et les «contre〉 de ce test.» Si les deux cas de figure déclinent de manière différente la confiance du patient envers son médecin, de nombreux collègues se positionnent favorablement dans cette complémentarité de deux expertises: le patient est l'expert de ses valeurs et préférences et le médecin est l'expert de la connaissance et de la relation thérapeutique. Les organisateurs avaient décidé d'ouvrir leur congrès aux patients en leur offrant une large place, en les invitant à donner leur vision de la relation patient-médecin et de leur place dans la décision médicale.

En 2019, le symposium romand du Collège suisse de médecine de premier recours fera lui-aussi place aux patients! Cette dynamique ne fait finalement que quittancer la belle formule «Encouraging patients to ask questions» [1].

\section{Référence}

1 Judson TJ et al. Encouraging patients to ask questions - How to overcome «white-coat silence». JAMA. 2013;309:2325-6. 